\title{
Beraprost Sodium for Chronic Diabetic Foot Ulcer: A Randomized Controlled Trial in Thammasat University Hospital
}

\author{
Surajit Awsakulsutthi, MD, ${ }^{1}$ Kwanjit Punpho, BSN, MSN, ${ }^{2}$ \\ Jinpitcha Mamom, BSN, MSN, ${ }^{3}$ Pairat Baikrut, ${ }^{4}$ and Patcharee Yingchoorod ${ }^{4}$
}

\begin{abstract}
Background: Chronic diabetic foot ulcer has a high rate of healing failure. Beraprost sodium, oral form of prostaglandin $\mathrm{I}_{2}$ analogue, maybe used for increasing wound healing.

Objective: To compare the healing rate of chronic diabetic foot ulcer between Beraprost sodium and control groups.

Design: A single blind randomized trial.

Materials and Methods: Fifty non-healed chronic diabetic foot ulcer patients were randomized to be the study and control groups. Beraprost sodium was prescribed in the study group according to protocol.

Results: At 6th week follow up, median wound healing rate in the study group was significantly higher than in the control group with the rate of $88.1 \%$ and $33.3 \%$, respectively. Complete wound healing in the study group was also significantly higher than in the control group (48\%:8\%). In the study group, 9 patients (37.5\%) could be administered with recommended dose. Side effects were headache in 14 patients (58.3\%) and palpitation in one patient (4.2\%). One patient was discontinued for severe headache.

Conclusions: The use of Beraprost sodium in chronic diabetic foot ulcer patients significantly increases the wound healing rate. Headache was the common side effect.
\end{abstract}

Keywords: chronic diabetic foot ulcer, Beraprost sodium, $\mathrm{PG} \mathrm{I}_{2}$ analogue

\section{Introduction}

In Thailand, the estimated national prevalence of diabetes is about $9.6 \% .{ }^{1)}$ Chronic diabetic foot ulcer is one of the most common problems, with increased

${ }^{1}$ Department of Surgery, Faculty of Medicine, Thammasat University, Pathumthani, Thailand

${ }^{2}$ Advanced Practice Nurse (Medical-Surgical), Thammasat

University Hospital, Pathumthani, Thailand

${ }^{3}$ Advanced Practice Nurse (Medical-Surgical),Faculty of

Nurse, Thammasat University, Pathumthani, Thailand

${ }^{4}$ Wound care team, Surgery department, Thammasat

University Hospital, Pathumthani, Thailand

Received: September 26, 2013; Accepted: January 20, 2014 Corresponding author: Surajit Awsakulsutthi, MD. Department of Surgery, Faculty of Medicine, Thammasat University (Rang-sit campus), Klonglaung, Pathumthani 12100, Thailand Tel: +66-2-926-9523, Fax: +66-2-926-9530

E-mail: awsakul@yahoo.com risk of lower limb amputation up to $22.1 \%^{2)}$ despite many wound treatment strategies.

The presence of prostaglandin $\mathrm{I}_{2}\left(\mathrm{PG}_{2}\right)$ analogue drug, Beraprost sodium (BPS), which has anti-platelet aggregation and vasodilatation effects, is efficient in treatment of peripheral arterial occlusive disease (PAOD). ${ }^{3)}$

In diabetes, BPS improved blood circulation ${ }^{4)}$ and ameliorates diabetic neuropathy in lower extremities. ${ }^{5,6)}$ In an animal study, BPS improved serum glucose in rats. ${ }^{7)}$ For these reasons, may BPS ameliorate healing of lower extremities wounds in diabetes?

Uncontrolled trial of BPS in diabetic gangrenous wounds had a beneficial effect in the healing process. ${ }^{8)}$ May BPS ameliorate wound healing in chronic diabetic foot ulcers?

Therefore, this study was done to determine the wound healing between BPS and the control group 
in patients with chronic diabetic foot ulcers. It was designed as a randomized controlled trial with the alternative hypothesis of BPS significantly increasing the rate of wound healing compared with control groups.

\section{Objectives}

Beraprost sodium, oral form PG $\mathrm{I}_{2}$ analogue, can be used for increasing healing rate of chronic diabetic foot ulcer.

\section{Materials and Methods}

The protocol was approved by the ethics committee of Thammasat University Hospital and Faculty of Medicine, Thammasat University prior to the commencement of this study (MTU-I-1-44/51). During June 2009 to July 2012, 50 chronic diabetic foot ulcer patients in the surgical department of Thammasat university hospital were enrolled in this study.

The inclusion criteria are male and female, more than 20 years old, with established diagnosis of diabetes mellitus by "DM clinic of Thammasat University Hospital", and have unhealed foot ulcers more than 3 weeks after proper management in the diabetes foot care clinic. The patients would be excluded if she/he have PAOD (ankle brachial index of less than 0.9 ), history of deep vein thrombosis, pregnant, bleeding abnormality (i.e. upper gastrointestinal ulcer, urinary bleeding, hemoptysis, vitreous body bleeding), active wound (i.e. wet gangrene, osteomyelitis), on drugs that might be interfere (i.e. vasodilators, anticoagulant), history of $\mathrm{PGI}_{2}$ drug allergy or hypersensitivity, abnormal of pre-study test such as coagulogram, bleeding time, liver function, or serum creatinine more than $2 \mathrm{mg} / \mathrm{dL}$.

All patients gave their written informed consent. The history of diabetic treatment, fasting blood sugar, $\mathrm{HbA1c}$, liver function test, serum creatinine and wound onset were recorded. The examination was to determine whether dorsalis pedis and posterior tibia arteries were palpable and to rule out the presence of an active infection. The investigations were plain film X-ray and ankle brechial pressure index $(\mathrm{ABI})$.

For statistical analysis reasons, only subjects that had one wound were selected.

\section{Randomized Allocation}

The study was designed as a single blind, randomized controlled trial. Eligible patients were allocated randomly into control or study groups by concealed envelopes. The study group received BPS; the control group received a placebo.

The local wound care of both groups was performed with normal saline cleansing and the application of Intrasite ${ }^{\circledR}$ gel (Smith \& Nephew Corp., London, UK) daily by the same well-trained nurse. The wounds were measured and calculated by the Smith \& Nephew wound measurement device (Visitrak ${ }^{\circledR}$; Smith \& Nephew Corp., London, UK) at 2nd, 4th and 6th week follow up. Both the nurse and measurer were blinded to the type of groups.

In the study group, following safety profiles, blood chemical tests such as serum glutamic oxaloacetic transaminase (SGOT)/ serum glutamic pyruvic transaminase (SGPT), coagulogram, and bleeding time were performed every 2 weeks. Electrocardiogram (EKG) was performed at initial and at the 2 nd week follow up.

The study group was initially administered with BPS $120 \mu \mathrm{g} /$ day ( $40 \mu \mathrm{g}$ oral three times per day) for the first week. If there was a minor side-effect, the dose was decreased to $60 \mu \mathrm{g} /$ day $(20 \mu \mathrm{g}$ oral three times per day) until 6 weeks. If there were no sideeffects, the dose of $120 \mu \mathrm{g} /$ day was used until 6 weeks.

The discontinuation criteria were when she/he wanted to discontinue treatment, active infection celebration or osteomyelitis detection or massive tissue necrosis, unstable vital signs, or drug side-effects (such as nausea, vomiting, abdominal pain, diarrhea, hypotension: systolic pressure less than $100 \mathrm{mmHg}$, myocardial infarction, raising liver function enzyme: SGOT or SGPT more than $10 \%$ )

The control group was administered a placebo and wound measurement was done at the $2 \mathrm{nd}$, 4th and 6 th week follow up.

\section{Statistical Analysis}

The baseline characteristics of the patients were described using mean and standard deviation or median and $75 \%$ interquartile range according to the distribution of the data for continuous data and frequency for categorical data. 
Table 1 Baseline characteristics of patients

\begin{tabular}{lccc}
\hline Variables & Study $\mathrm{n}=24$ & Control $\mathrm{n}=25$ & $\mathrm{p}$ value \\
\hline Age, mean (SD) & $60.9(11.36)$ & $58.2(11.42)$ & 0.40 \\
Sex, $\mathrm{n}(\%)$ & & & \\
Male & $13(54.2)$ & $9(36.0)$ & 0.20 \\
Female & $11(45.8)$ & $16(64.0)$ & \\
Smoking & $1: 23$ & $0: 25$ & $0.49^{*}$ \\
FBS, mean (SD) & $162.88(70.5)$ & $160.32(53.6)$ & 0.89 \\
$\mathrm{HbA}{ }_{1}$ C, mean (SD) & $7.4(1.79)$ & $8.4(2.24)$ & 0.08 \\
DM treatment & & & $1.00^{*}$ \\
Insulin & 1 & 23 & 0.38 \\
Oral anti-DM & 23 & $1.01(0.16)$ & \\
ABI, mean (SD) & $1.05(0.13)$ & & \\
Initial wound size & $3.4(2.0)$ & $3.1(3.1)$ & $0.49^{* *}$ \\
Mean (SD) & $3.0(2.0,5.2)$ & $1.0,6.4)$ & \\
Median (75\% IQR) & & &
\end{tabular}

*: Fisher exact test; **: Wilcoxon rank-sum test (Mann-Whitney); FBS: fasting blood sugar; DM: diabetes mellitus; $\mathrm{ABI}$ : ankle brechial pressure index; IQR: interquartile range; $\mathrm{SD}$ : standard deviation

Table 2 Side-effect incident in study groups

\begin{tabular}{ll}
\hline Monitor & Incident \\
\hline - EKG & - \\
- SGOT/SGPT & - \\
- INR & - \\
- Bleeding time & - \\
- Side effect & \\
- headache & $14 / 24$ \\
- palpitation & $1 / 24$ \\
\hline EKG: electrocardiogram; SGOT: serum glutamic oxalo- \\
acetic transaminase; SGPT: serum glutamic pyruvic \\
transaminase; INR: international normalized ratio
\end{tabular}

Patient baseline characteristics were compared between the study groups, using $\chi^{2}$ test or Fisher's exact test, and student t-test or Wilcoxon rank-sum test (Mann-Whitney) were used for categorical data and continuous data, respectively as appropriated. Healing rate at 6 weeks was compared between study groups with the Wilcoxon rank-sum test. If unbalanced baseline characteristics between groups occurred, then multivariate analysis would be performed to adjust its effect. p-value of less than 0.05 was considered to be statistically significant.

\section{Results}

There were 25 patients in the study group and 25 patients in the control group. One patient in the study group was removed from the study for side effect of severe headache. In the remaining study group were 13 males and 12 females, with a mean age of 60.9 years (range: 41-82). In the control group were nine males and 16 females, with a mean age of 58.2 years (range: 33-78). All patients of both groups had active pulsation of dorsalis pedis and posterior tibia arteries.

Baseline characteristics of patients between the two groups (i.e. age, sex and initial wound size) were not significantly different (Table 1 ).

In the study group, 9 patients $(37.5 \%)$ could be administered with BPS $40 \mu \mathrm{g}$ oral three times per day for 6 weeks. Side effects were headache in fourteen patients $(58.3 \%)$ and palpitation in one patient $(4.2 \%)$, but all could be administered with BPS $20 \mu \mathrm{g}$ oral three times per day until 6 weeks. No abnormal changes were noticed in EKG, SGOT/SGPT, coagulogram, or bleeding time in any patients (Table 2 ).

Comparing the study and control groups, the rate of wound healing at $2 \mathrm{nd}, 4 \mathrm{th}$, 6 th week in the study group were $35.6 \%, 67.8 \%, 85.8 \%$, respectively (Fig. 1); and in the control group were $20.1 \%, 30.3 \%, 41.5 \%$, respectively (Table 3 ). At the 6 th week, the median wound healing rate in the study group was significantly higher than the wound healing rate of the control group with a p-value of less than 0.001 . Complete wound healing in the study group was also significantly higher than in the control group with a p-value of less than 0.001 (Table 4).

\section{Discussion}

$\mathrm{PGI}_{2}$ actions are anti-platelet aggregation, vasodilatation, and endothelial function improvement. ${ }^{9)}$ The mechanisms of action are believed to act at the $\mathrm{PGI}_{2}$ receptor of platelet and endothelial smooth muscle cells. Problems in the clinical application of $\mathrm{PGI}_{2}$ are 


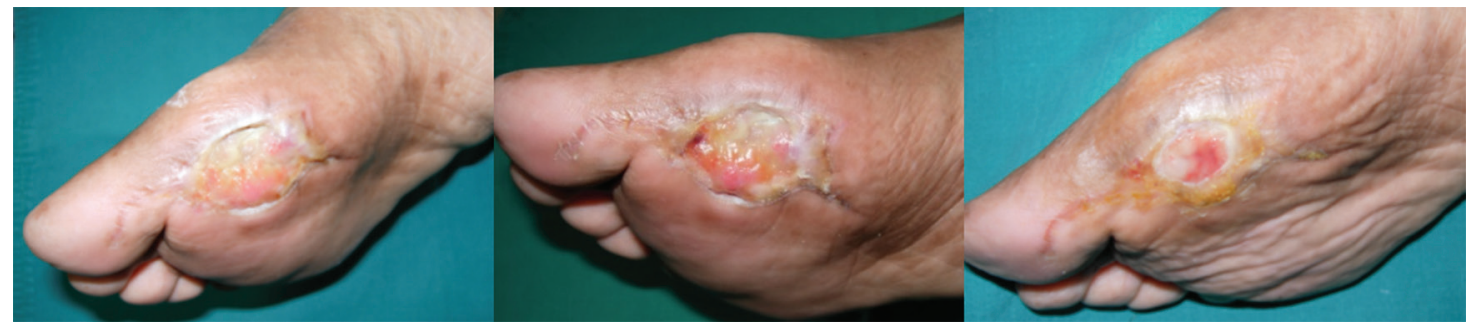

Fig. 1 Wound healing after used beraprost sodium at 0, 2nd, 6th week.

Table 3 Results of rate of wound healing (\%)

\begin{tabular}{lcc}
\hline Post-treatment & Study $n=24$ & Control $n=25$ \\
\hline 2nd week & 35.6 & 20.1 \\
4th week & 67.8 & 30.3 \\
6th week & 85.8 & 41.5 \\
\hline
\end{tabular}

the chemical instability with a short plasma half-life of approximately $3 \mathrm{~min},{ }^{10)}$ and severe adverse reactions such as hypotension, facial hot flushes, headache etc. due to its potent vasodilative action.

Iloprost is developed as a $\mathrm{PGI}_{2}$ analogue that is chemically stable and used in an intravenous form for treatment in peripheral arterial insufficiency patients. ${ }^{11)}$ It can reduce claudication symptoms and increase the wound healing rate.

BPS is another developed chemically stable $\mathrm{PGI}_{2}$ analogue used in oral form. ${ }^{12)}$ With weak hypotensive action it resolved the severe adverse reactions problems. From 1992 BPS has been used in treatment of primary pulmonary hypertension. ${ }^{13)}$

Since 1996, many trial studies of BPS in chronic obstructive arterial disease and diabetes mellitus were reported. ${ }^{14,15)}$

In 1998, trial treatment of chronic obstructive arterial disease with BPS, resulted in improvements of rest pain and coldness of limbs $60.9 \%$ and ischemic ulcer size $69.6 \%$ and increased granulation was $82.6 \% .{ }^{16)}$

In 1999, research in ASO patients complicated with diabetes mellitus, BPS improved leg blood flow. ${ }^{17)}$

In 2001, research in diabetes mellitus patients, resulted in significantly increased skin blood flow in all ABI level for non-DM patients and DM patients. ${ }^{4)}$

In 2002, research in DM patients with PAOD, resulted in significantly increased ABI, and symptoms such as coldness, numbness in lower extremities significantly improved.5)
In 2010, obese Zucker rats, an experimental model of genetic obesity-induced type 2 diabetes, BPS improved serum glucose and significant increase in temperature of the hind limb, which was likely due to improved blood circulation. ${ }^{7)}$

In the 2010 first Asian PAD workshop, the beneficial effect of BPS in the healing process of diabetic gangrenous wounds was presented. ${ }^{8)}$

In the 2013 uncontrolled study, BPS was the effective treatment for improving various subjective symptoms in the lower extremities, such as burning, coldness, edema, exertional pain, stabbing, and paresthesia, in diabetic patients with peripheral arterial disease. $^{6)}$

In this study, the author designed a randomized controlled trial for studying the result of wound size healing and side-effects using the treatment dose of BPS in chronic diabetic foot ulcer patients that were not in an active ischemic leg. The recommended dose was $120 \mu \mathrm{g} / \mathrm{day}$, but the study revealed that many patients in the study group had side-effects such as headache. So for safety even if the patient complained about only minor headache, the dose of $60 \mu \mathrm{g} / \mathrm{day}$ was used.

Nine patients $(37.5 \%)$ in the study group could use the recommended dose of $120 \mu \mathrm{g} / \mathrm{day}$. The complaint of headache was common $(58.3 \%)$ but most patients could tolerate a dose of $60 \mu \mathrm{g} / \mathrm{day}$, except one that was discontinued. One male patient complained about palpitation, and EKG and cardiac function tests were performed with results showing no abnormal patterns. The symptom did not recur when he received BPS $60 \mu \mathrm{g} / \mathrm{day}$.

The study revealed that the wound healing rate of diabetic foot ulcer patients in the study group was significantly higher than in the control group. The blood chemical tests such as SGOT/SGPT, coagulogram, bleeding time, and EKG were not affected in both study groups. 
Table 4 Wound healing rate at 6 th week

\begin{tabular}{lccc}
\hline Healing rate & Study $n=24$ & Control $n=25$ & p value \\
\hline $\begin{array}{l}\text { Healing rate (\%) } \\
\text { (Median, 75\% IQR) }\end{array}$ & $88.1(64.3,100)$ & $33.3(7.7,61.8)$ & $<0.001^{*}$ \\
Heal & $12(48 \%)$ & $2(8 \%)$ & $<0.001$ \\
- Complete & $13(52 \%)$ & $23(92 \%)$ & \\
- Not complete & & & \\
\hline
\end{tabular}

* Wilcoxon rank-sum test (Mann-Whitney). IQR: interquartile range

Diabetic vascular disease refers to the development of blockages in the arteries, sometimes called "hardening of the arteries", throughout the body. The macro-vascular manifestations include atherosclerosis and medial calcification. ${ }^{18,19)}$ Fatty deposits and platelet aggregation build up in the inner linings of the artery walls of the legs and hinder blood flow. Partial arterial occlusion in multiple sites often presents in a diabetic foot, which causes decreasing vascularization and a slowing of wound healing. Arterial pathophysiology of diabetic foot ulcer is more peripheral, in which re-vascularization surgery often cannot be performed. Many patients do not have an active ischemic leg and some patients are not suitable for re-vascularization surgery. The aim of the study is a trial of alternative effective medical treatment that achieves wound healing improvement in non-healed diabetic foot ulcer patients.

How does BPS improve wound healing? Aside from increasing blood flow with anti-platelet aggregation, vasodilatation and endothelial function improvement, ${ }^{20)}$ BPS improves serum glucose ${ }^{7)}$ which improves cell function in the healing process and ameliorates diabetic neuropathy. ${ }^{5,6,21)}$ This results in an increased healing rate as seen in our study.

With cost-effectiveness consideration, these advantages would benefit non-healed chronic diabetic foot ulcer patients who do not need re-vascularization surgery or are not in a condition for which it can be performed.

\section{Conclusions}

The use of Beraprost sodium, oral form $\mathrm{PGI}_{2}$ analogue, in chronic diabetic foot ulcer patients can significantly increase the wound healing rate. Headache was the common side effect in recommended dose.

\section{Acknowledgement}

The authors would like to extend their grateful thanks to Boonying Siribumrungwong, Faculty of Medicine, Thammasat University for statistical consulting and all paramedical officers in out-patient surgery unit of Thammasat University Hospital.

\section{Disclosure Statement}

The authors reported no potential conflicts of interest relevant to this article.

\section{References}

1) Aekplakorn W, Stolk RP, Neal B, et al. The prevalence and management of diabetes in Thai adults: the international collaborative study of cardiovascular disease in Asia. Diabetes Care 2003; 26: 2758-63.

2) Krittiyawong S, Ngarmukos C, Benjasuratwong Y, et al. Thailand diabetes registry project: prevalence and risk factors associated with lower extremity amputation in Thai diabetics. J Med Assoc Thai 2006; 89 Suppl 1: S43-8.

3) Nishibe T. Beraprost: optimising drug therapy for PAD with complications. Ann Vasc Dis 2010; 3: 97-8.

4) Aso Y, Tayama K, Takanashi K, et al. Changes in skin blood flow in type 2 diabetes induced by prostacyclin: association with ankle brachial index and plasma thrombomodulin levels. Metab Clin Exp 2001; 50: 568-72.

5) Toyota T, Oikawa S; Beraprost Sodium Study Group. Effects of beraprost sodium (Dorner) in patients with diabetes mellitus complicated by chronic arterial obstruction. Angiology 2002; 53: 7-13.

6) Yoon HS, Choi WJ, Sung IH, et al. Effects of Beraprost sodium on subjective symptoms in diabetic patients with peripheral arterial disease. Clin Orthop Surg 2013; 5: 145-51.

7) Sato N, Kaneko M, Tamura M, et al. The prostacyclin analog beraprost sodium ameliorates characteristics of metabolic syndrome in obese Zucker (fatty) rats. Diabetes 2010; 59: 1092-100. 
8) Puruhito. Efficacy of beraprost among ambulatory treated patients with diabetic angiopathy with gangrenous wounds-experience from Surabaya, Indonesia. Ann Vasc Dis 2010; 3: 93.

9) Vane JR, Botting RM. Pharmacodynamic profile of prostacyclin. Am J Cardiol 1995; 75: 3A-10A.

10) GlaxoWellcome. Epoprostenol prescribing information (online). Available from http://www.flolan-center.com/ pages/flolan (Accessed $2001 \mathrm{March}$ ).

11) Fiessinger JN, Schäfer M. Trial of iloprost versus aspirin treatment for critical limb ischaemia of thromboangiitis obliterans. The TAO Study. Lancet 1990; 335: $555-7$.

12) Toda N. Beroprot sodium. Cardiovasc Drug Rev 1988; 6: 222-38.

13) Brij S, Peacock AJ. Pulmonary hypertension: its assessment and treatment. Thorax 1999; 54 Suppl 2: S28-32.

14) Aoki T, Yamaguchi T, Ueda N, et al. Effect of oral PG $I_{2}$ derivative on peripheral circulation. Geriatr Med 1995; 34: 129-33.

15) Goya K, Otsuki M, Xu X, et al. Effects of the prostaglandin $\mathrm{I}_{2}$ analogue, beraprost sodium, on vascular cell adhesion molecule- 1 expression in human vascular en- dothelial cells and circulating vascular cell adhesion molecule-1 level in patients with type 2 diabetes mellitus. Metab Clin Exp 2003; 52: 192-8.

16) Suh BY, Kwun KB, Kwun TW, et al. Open-label, uncontrolled, 6-week clinical trial of beraprost in patients with chronic occlusive arterial disease. Curr Ther Res 1998; 59: 645-53.

17) Toyota T, Oikawa S, Satoh T, et al. Efficacy of beraprost sodium against chronic arterial obstruction in patients with concomitant diabetes. Endocrinology \& Diabetology 1999; 8: 104-18.

18) Libby P. Changing concepts of atherogenesis. J Intern Med 2000; 247: 349-58.

19) Nomura S, Shouzu A, Omoto $S$, et al. Significance of chemokines and activated platelets in patients with diabetes. Clin Exp Immunol 2000; 121: 437-43.

20) Kubota T, Kubota N, Kumagai H, et al. Impaired insulin signaling in endothelial cells reduces insulin-induced glucose uptake by skeletal muscle. Cell Metab 2011; 13: 294-307.

21) Shin S, Kim KJ, Chang HJ, et al. The effect of oral prostaglandin analogue on painful diabetic neuropathy: a double-blind, randomized, controlled trial. Diabetes Obes Metab 2013; 15: 185-8. 